\title{
Regional Thermodynamic Characteristics Distinguishing Long- and Short-Duration Freezing Rain Events over North America
}

\author{
Christopher D. McCray, John R. Gyakum, and Eyad H. Atallah ${ }^{\mathrm{a}}$ \\ Department of Atmospheric and Oceanic Sciences, McGill University, Montreal, Quebec, Canada
}

(Manuscript received 26 August 2019, in final form 24 January 2020)

\begin{abstract}
Freezing rain is an especially hazardous winter weather phenomenon that remains particularly challenging to forecast. Here, we identify the salient thermodynamic characteristics distinguishing long-duration (six or more hours) freezing rain events from short-duration (2-4 h) events in three regions of the United States and Canada from 1979 to 2016. In the northeastern United States and southeastern Canada, strong surface coldair advection is not common during freezing rain events. Colder onset temperatures at the surface and in the near-surface cold layer support longer-duration events there, allowing heating mechanisms (e.g., the release of latent heat of fusion when rain freezes at the surface) to act for longer periods before the surface reaches $0^{\circ} \mathrm{C}$ and precipitation transitions to rain. In the south-central United States, cold air at the surface is replenished via continuous cold-air advection, reducing the necessity of cold onset surface temperatures for event persistence. Instead, longer-duration events are associated with warmer and deeper $>0^{\circ} \mathrm{C}$ warm layers aloft and stronger advection of warm and moist air into this layer, delaying its erosion via cooling mechanisms such as melting. Finally, in the southeastern United States, colder and especially drier onset conditions in the cold layer are associated with longer-duration events, with evaporative cooling crucial to maintaining the subfreezing surface temperatures necessary for freezing rain. Through an improved understanding of the regional conditions supporting freezing rain event persistence, we hope to provide useful information to forecasters in their attempt to predict these potentially damaging events.
\end{abstract}

\section{Introduction}

One of the greatest challenges forecasters face during the winter is predicting the phase of precipitation (e.g., Ralph et al. 2005). Forecasting freezing rain is particularly difficult, as it forms under very similar conditions to ice pellets yet is considerably more hazardous. Freezing rain may form through one of two mechanisms: the melting process or the supercooled warm rain process. The melting process is characterized by a layer of $>0{ }^{\circ} \mathrm{C}$ air aloft (the warm layer) with subfreezing layers above and below it (e.g., Brooks 1920; Meisinger 1920). Snow initially forms aloft and then melts upon descent through this warm layer. The resultant raindrops fall into the $\leq 0^{\circ} \mathrm{C}$ cold layer and finally refreeze upon contact with

\footnotetext{
${ }^{\text {a }}$ Current affiliation: Department of Hydrology and Atmospheric Sciences, The University of Arizona, Tucson, Arizona.
}

Corresponding author: Christopher D.McCray, christopher.mccray@ mail.mcgill.ca subfreezing surfaces. This refreezing can result in extremely hazardous conditions, as the accreted ice can make surfaces slippery and weigh down and break trees and power lines (e.g., DeGaetano 2000; Changnon 2003).

The supercooled warm rain process also results in ice accretion at the surface, but does not require a $>0{ }^{\circ} \mathrm{C}$ warm layer (Bocchieri 1980; Huffman and Norman 1988; Rauber et al. 2000). Precipitation forms not as snow, but instead as rain or drizzle via collision and coalescence. This typically occurs with upward motion in shallow, low-level saturated layers lacking active ice nuclei. This formation mechanism most commonly produces freezing drizzle, though Rauber et al. (2000) also identified several freezing rain cases in which the supercooled warm rain process was likely occurring.

Stewart (1985) described the self-limiting nature of freezing rain, in that the diabatic processes within the warm and cold layers act to erode these layers in the absence of compensatory mechanisms. In the warm layer, cooling via the extraction of latent heat of fusion as snowflakes melt develops isothermal $0^{\circ} \mathrm{C}$ layers from 
the top down (Wexler et al. 1954; Stewart 1985; Kain et al. 2000). At the surface, the freezing of raindrops releases latent heat of fusion, which can warm the cold layer to $0^{\circ} \mathrm{C}$ (e.g., Lackmann et al. 2002). Processes such as warm-air advection (WAA) in the warm layer and cold-air advection (CAA) or evaporative cooling in the cold layer are necessary to offset these selflimiting effects for prolonged freezing rain to occur (e.g., Lackmann 2011). As a result, freezing rain events tend to be short-lived, with 1-h events most common and an exponential decrease in frequency with increasing duration (Cortinas et al. 2004; McCray et al. 2019).

Noteworthy ice storms tend to be those that deviate from these typically brief durations. During the extremely damaging 1998 ice storm in southeastern Canada and the northeastern United States, for example, freezing rain fell over a 5-day period at some locations (DeGaetano 2000; Gyakum and Roebber 2001; Roebber and Gyakum 2003; Henson et al. 2007). A key question arising from such cases is the following: What thermodynamic conditions allow freezing rain to persist for many hours, despite the diabatic processes acting to destroy the necessary temperature profile?

Several studies have examined environments conducive to freezing rain over various regions, primarily focusing on severe/prolonged events, and identified several mechanisms that help sustain these events (e.g., Cortinas 2000; Rauber et al. 2001; Robbins and Cortinas 2002; Ressler et al. 2012; Castellano 2012; Sanders et al. 2013; Mullens et al. 2016b). For example, the cold layer can be maintained via low-level CAA, which is sometimes enhanced through local terrain impacts on the near-surface winds (Ressler et al. 2012; Sanders et al. 2013; Mullens et al. 2016b). The warm layer is often sustained via midlevel WAA poleward of a warm or quasi-stationary front (Ressler et al. 2012; Castellano 2012; Mullens et al. 2016b).

One method to identify conditions supporting severe freezing rain events is to compare the characteristics of severe and weak cases. This type of comparison is complicated by the lack of consistent observations of ice accretion, particularly for weaker events. For example, several prior studies (Rauber et al. 2001; Castellano 2012; Sanders et al. 2013; Mullens et al. 2016b) identified events using NOAA's Storm Data product, which is based on public reports of significant weather events and damage and is therefore unlikely to contain lesssevere cases.

In McCray et al. (2019), we examined freezing rain events at surface stations in the United States and Canada and used event duration as a proxy for severity, with long-duration (LD) events being those with $\geq 6 \mathrm{~h}$ of freezing rain. Our climatology of these events highlighted

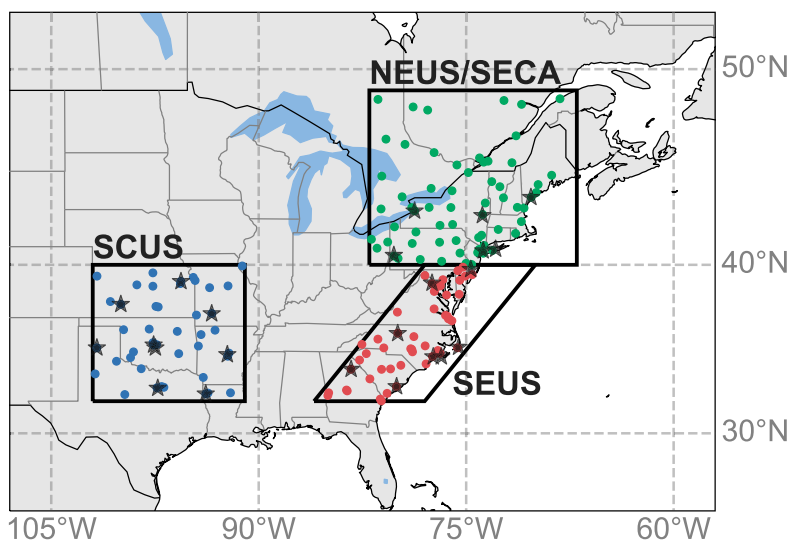

FIG. 1. Focus regions discussed in the text, with surface stations (dots) and upper-air sites (stars) within each plotted (from McCray et al. 2019, their Fig. 2). Regions include the northeastern United States/southeastern Canada (NEUS/SECA), the south-central United States (SCUS), and the southeastern United States (SEUS).

three focus regions (Fig. 1) where freezing rain and/or LD events are relatively common compared with other regions. Freezing rain and $\mathrm{LD}$ events are most common in the northeastern United States/southeastern Canada (NEUS/SECA), with 1-3 LD events $\mathrm{yr}^{-1}$ at most stations. In the south-central United States (SCUS), LD events are more rare, but freezing rain events of extremely long-duration ( $\geq 18 \mathrm{~h}$, the 99th percentile of duration among all events in the database) have occurred often there relative to other regions. Finally, the southeastern United States (SEUS) region represents the southward extension of relatively frequent LD events east of the Appalachian Mountains, in which $\geq 50 \%$ of events are LD at many stations.

Using surface and upper-air observations, we identified the archetypal thermodynamic evolution of LD events in each of these regions. In the NEUS/SECA, surface temperatures at onset are the coldest of the three regions. The warm layer develops via strong WAA aloft, and warming at the surface occurs through the release of latent heat of fusion as rain freezes. Lacking strong low-level CAA to sustain the cold layer, surface temperatures typically rise during events until the surface reaches $0^{\circ} \mathrm{C}$, causing precipitation to transition to rain. In the SCUS, however, the near-surface cold air is generally sustained by CAA and evaporative cooling supported by dry-air advection, resulting in particularly prolonged events. There, events most commonly end in either no precipitation or frozen precipitation as the warm layer dries and erodes. In the SEUS, onset cold layers are especially dry compared to the other regions, with evaporative cooling and weak surface CAA usually sustaining subfreezing surface temperatures during 
persistent freezing rain. Still, events most often end there once the surface temperature reaches $0^{\circ} \mathrm{C}$ and precipitation transitions to rain. While all possible phase evolutions have occurred in each of these regions, we found a clear propensity for the aforementioned transitions in each of them.

The results of McCray et al. (2019), motivated by past studies (e.g., Stewart 1985; Stewart and King 1987; Cortinas 2000; Kain et al. 2000; Lackmann et al. 2002), lead to several hypotheses regarding the conditions potentially supporting prolonged events in each of these regions:

1) In the NEUS/SECA and SEUS, longer-duration freezing rain, compared with less-persistent events, may occur when onset surface temperatures are colder (several degrees $<0^{\circ} \mathrm{C}$ ), allowing heating mechanisms to be sustained for longer periods before the surface reaches $0^{\circ} \mathrm{C}$ and precipitation transitions to rain.

2) In the SCUS, a particularly warm/deep onset warm layer may postpone erosion of the layer and allow events to persist for a longer period before precipitation transitions to snow/ice pellets. Similarly, nearsaturated onset warm layers could delay drying of the layer and the cessation of precipitation or transition to freezing drizzle.

3) In the SEUS, drier onset conditions at the surface and in the cold layer may support increased/more prolonged evaporative cooling, which helps to sustain the cold layer and support longer-duration events there.

The purpose of this paper is to test these hypotheses and to determine key thermodynamic characteristics differentiating persistent freezing rain events from brief ones in each of the three focus regions. These results could help focus forecaster attention on particular fields essential to producing a severe event in their region of interest.

\section{Data and methods}

\section{a. Event identification and surface data}

We identify freezing rain events using the dataset of surface observations developed in McCray et al. (2019) using NOAA's Integrated Surface Database (Smith et al. 2011). This dataset includes all hourly observations of freezing rain, including mixtures with other precipitation types, at 579 U.S. and Canadian surface stations from 1979 to 2016. Events are identified by first grouping consecutive hourly observations of freezing rain at each station, and then combining events that are separated by fewer than $24 \mathrm{~h}$ such that events are, roughly, synoptically independent. The event duration is the number of hourly freezing rain observations between and including the onset and end times, and excluding the hours between onset and end during which freezing rain was not observed. Observations of freezing drizzle are not included in the dataset, though it is possible that some freezing drizzle observations were erroneously reported as freezing rain. Additional details, including qualitycontrol methods used to select the included stations, are discussed in McCray et al. (2019, section 2).

We identify LD events as those in which six or more hours of freezing rain were observed. This threshold is consistent with past studies (Cortinas 2000; Ressler et al. 2012) and produces a set of cases that are relatively rare (only $20 \%$ of events in the dataset) but still provides a sufficiently large sample to examine. The severity of a freezing rain event is most closely related to total ice accretion. However, freezing rain is typically of light intensity (Cortinas 2000; Ressler et al. 2012; McCray et al. 2019) and ice accretes less efficiently when precipitation is heavier, with increased runoff than during lighter freezing rain (e.g., Sanders and Barjenbruch 2016).

In 2013, some ASOS (NOAA 1998) stations in the United States began reporting hourly ice accretion calculated using the icing sensor (Ryerson and Ramsay 2007). In our dataset, ice accretion data were available for 1321 events at 211 U.S. stations between 2013 and 2016. We calculate total ice accretion for each case by summing hourly accretion totals during the event and do the same for ASOS precipitation amounts. Among these events, total ice accretion correlates more strongly with event duration $(r=0.76)$ than with total precipitation $(r=0.60)$. Given the limited spatial and temporal availability of ice accretion data and, to a lesser extent, precipitation amount data, duration therefore provides a strong proxy for ice accretion and event severity that can be calculated for any station reporting freezing rain.

We compare LD cases with short-duration (SD) events, during which $2-4 \mathrm{~h}$ of freezing rain were observed. One-hour events are excluded, as the uncertainty in the timing of radiosonde observations makes it difficult to ascertain at what time relative to event onset the observation was taken. ${ }^{1}$ We also exclude the intermediate 5-h cases to allow for a clearer separation

\footnotetext{
${ }^{1}$ For example, a 1200 UTC radiosonde is likely to have been released between 1100 and 1200 UTC. A 1-h freezing rain event observed at 1100 UTC may have ended at any point within that hour as well. It is thus impossible to determine whether the radiosonde observation is representative of the environment before, during, or after freezing rain during these cases.
} 
between the SD and LD events. Various event definitions were tested, and while quantitative results vary based on the thresholds chosen, inclusion of 1 - or 5 -h events does not change the qualitative results presented here.

Upon identification of events, we compare surface observations at the onset of the two categories, in particular temperature and dewpoint depression $T_{\mathrm{DD}}$ and the evolution of precipitation phase preceding and following events in each region. The U.S. stations that were automated during the implementation of the ASOS network in the 1990s are capable of detecting freezing rain (NOAA 1998), and report it with similar frequencies to manual stations (Reeves 2016). We are therefore confident in the inclusion of these stations in our dataset. However, ASOS stations cannot detect ice pellets or freezing drizzle (NOAA 1998), which are similar to and often occur surrounding freezing rain events. Thus, we limit examination of phase changes to observations of events prior to 1995 . For all other metrics, we examine observations over the full 1979-2016 period.

\section{b. Upper-air observations}

In McCray et al. (2019), we compared thermodynamic profiles at freezing rain event onset and end to explore the evolution of LD events. Here, we perform a similar analysis, instead comparing profiles observed at LD onset with those taken at SD onset. Upon identification of SD and LD events, we examine radiosonde observations using the University of Wyoming archive (available online at http://weather.uwyo.edu/upperair/ sounding.html). Developing a representative sample of radiosonde observations is challenging, as freezing rain is relatively uncommon compared with other precipitation types (e.g., Cortinas et al. 2004) and soundings are typically only available twice daily. To overcome this, we examine observations at all stations within a given focus region together. While variation within each region is to be expected, the thermodynamic profiles observed during freezing rain in the given regions are fairly coherent, particularly when comparing between regions (McCray et al. 2019).

We search for soundings for SD and LD events whose onset occurred within one hour of 0500,1100 , 1700 , or 2300 UTC, accounting for the fact that radiosondes are typically released $30-60 \mathrm{~min}$ prior to the synoptic time $(0000,1200$, and, less commonly, 0600 or 1800 UTC). An additional challenge is that freezing rain commonly occurs in narrow, elongated bands (e.g., Rauber et al. 2001; Changnon 2003), with precipitation phase varying on either side of the band. Thus, slight temporal and spatial offsets between the surface observation and its associated sounding as well as drift of the radiosonde as it ascends can make a sounding unrepresentative of the freezing rain environment. We therefore limit our search to sounding sites located within a $40-\mathrm{km}$ radius of one of the surface stations in our dataset, following the methodology of Reeves et al. (2014). We add an additional criterion that surface and upper-air sites be within 100-m elevation of each other.

The maximum distance between a surface and upperair site among the stations meeting these criteria is $22 \mathrm{~km}$, and only 4 of the 21 sites are $\geq 10 \mathrm{~km}$ apart. We tested various temporal and spatial criteria to determine their impacts on our results. Stricter distance or time criteria reduced sample sizes while not changing our qualitative results. Though we cannot be certain what type of precipitation was falling at the precise time and location of the radiosonde measurement, we believe the examination of grouped data from many events and stations within each focus region allows us to identify salient features representative of freezing rain in each.

We identify the warm layer as the layer aloft through which the temperature $T>0^{\circ} \mathrm{C}$. The warm layer must be located above a cold layer, which is defined as the nearsurface layer through which $T \leq 0^{\circ} \mathrm{C}$. We then calculate several metrics for these layers, including the depth and maximum temperature $T_{\max }$ of the warm layer and the depth and minimum temperature $T_{\min }$ of the cold layer.

In some instances, a warm layer is absent, possibly due to the radiosonde sampling the environment just before or after freezing rain, or because freezing rain was produced via the supercooled warm rain process. In these cases, we cannot identify the warm and cold layers and, therefore, the aforementioned parameters that describe them. To remedy this, we employ two additional parameters: the maximum temperature in the 250-2500 m above ground level (AGL) layer $T_{\max }^{250-2500 \mathrm{~m}}$ and the minimum temperature in the $0-1000 \mathrm{~m}$ AGL layer $T_{\min }^{0-1000 \mathrm{~m}}$. These layers represent the approximate levels within which the warm-layer maximum and cold-layer minimum temperatures are commonly found when these layers are present. We retain the cases lacking a warm layer for our analysis and composites (Fig. 6), as even if not necessarily representative of conditions during freezing rain, these soundings do represent conditions near event onset.

As in McCray et al. (2019), we also examine cloud depth and cloud-top temperature in the soundings. Following the methodology of Rauber et al. (2000), we estimate cloud layers as those through which $T_{\mathrm{DD}}<3^{\circ} \mathrm{C}$, with the cloud top being the first level above the cloud layer where $T_{\mathrm{DD}}$ exceeds $3^{\circ} \mathrm{C}$ over a depth of at least $1 \mathrm{~km}$. Note that this is only an estimate of the cloud layer, and leaves open the possibility of additional cloud 
layers that could promote a seeder-feeder process (e.g., Rutledge and Hobbs 1983) whereby ice particles from upper layers serve as ice nuclei in a lower layer.

\section{c. Reanalysis data}

Finally, we calculate temperature and moisture advection during freezing rain events using the NCEP Climate Forecast System Reanalysis (CFSR; Saha et al. 2010) for 1979-2010 and its extension, the Climate Forecast System, version 2 (CFSv2; Saha et al. 2014), for 2011-16, both on a $0.5^{\circ}$ latitudelongitude grid. We calculate temperature advection $(-\mathbf{v} \cdot \nabla T)$ and moisture advection (advection of water vapor mixing ratio, $-\mathbf{v} \cdot \nabla w$ ) at the surface (advection of 2-m temperatures/mixing ratios by the $10-\mathrm{m}$ wind) and aloft at the level of $T_{\max }^{250-2500 \mathrm{~m}}$, equivalent to the level of warm-layer $T_{\max }$ when a warm layer is present. To reduce the possibility of erroneous data, especially in the vicinity of the complex terrain of the NEUS/SECA, we only calculate advection at the onset of events at times and locations at which we have observed soundings in the radiosonde dataset described above, as these upper-air data are assimilated in the CFSR. We smooth the calculated advection grids slightly using a Gaussian smoother (for details, see McCray et al. 2019) and then retrieve the value at the nearest-neighbor grid point to the surface station. An evaluation of the CFSR soundings (not shown) showed strong agreement with the observed soundings, lending confidence to the use of this reanalysis dataset for these calculations.

\section{Comparison of conditions at long- and short-duration event onset}

We now examine the hypotheses discussed in section 1 for each focus region through a comparison of the surface and upper-air conditions observed at the onset of SD and LD freezing rain events. We evaluate differences between the two event types using the two-sided Mann-Whitney $U$ test (e.g., Wilks 2011). Following the statement by the American Statistical Association (Wasserstein and Lazar 2016) and suggestions proposed by Hurlbert et al. (2019), we avoid discussion of "statistically significant" differences between the two categories based on a particular $p$-value threshold. We instead present the $p$ values themselves as well as visualizations (boxplots) of the distributions of each characteristic discussed.

\section{a. NEUS/SECA}

In McCray et al. (2019), we found that LD freezing rain events in the NEUS/SECA typically end in a transition

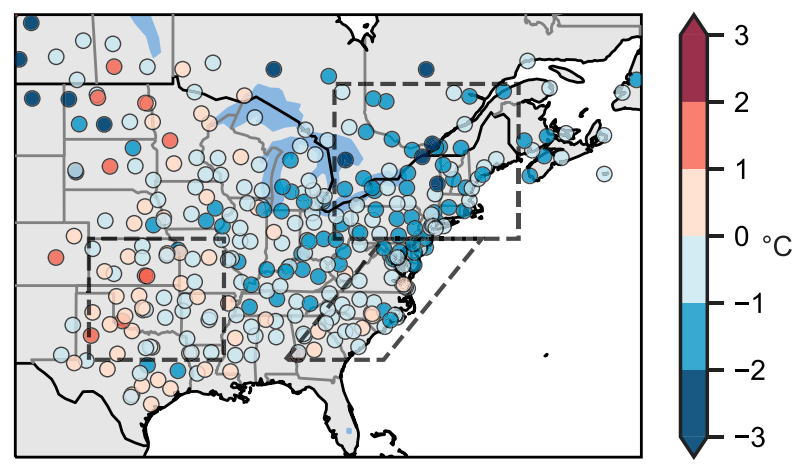

FIG. 2. Differences in mean onset surface temperatures between $\mathrm{SD}$ and $\mathrm{LD}$ events $\left({ }^{\circ} \mathrm{C}\right)$, calculated by taking the mean temperature observed at LD event onset minus the mean temperature observed at SD event onset at each station. Only stations where at least five SD and five LD events were observed are displayed. Dashed lines indicate the boundaries of each focus region.

from freezing rain to rain as surface temperatures reach $0^{\circ} \mathrm{C}$. Surface warming usually occurs throughout events due to the lack of strong surface CAA to offset the warming via latent heat release when rain freezes at the surface. This suggests a colder surface and colder/deeper cold layer at onset may allow for longer-duration events here.

To examine this possibility, we first calculate the mean onset surface temperature for LD events and SD events at each station and then take the difference between the two. The resulting map shows that among the three focus regions, coherent differences between onset temperatures for the two event types are strongest over the NEUS/SECA, where mean surface temperatures are $1^{\circ}-3^{\circ} \mathrm{C}$ colder at $\mathrm{LD}$ event onset than at $\mathrm{SD}$ event onset (Fig. 2). Distributions of onset temperatures among events at all stations in the NEUS/SECA show that SD onset temperatures are strongly skewed toward $0^{\circ} \mathrm{C}$ (Fig. 3a), with a regional mean (median) of $-3.0^{\circ} \mathrm{C}$ $\left(-2.7^{\circ} \mathrm{C}\right)$ for $\mathrm{LD}$ events and $-1.7^{\circ} \mathrm{C}\left(-1.0^{\circ} \mathrm{C}\right)$ for SD events $(p<0.001) .70 \%$ of LD events begin with surface temperatures $<-1.0^{\circ} \mathrm{C}$ compared with only $46 \%$ of SD events.

Increased potential for evaporative cooling when the near-surface air is particularly dry at onset may also help offset surface warming due to latent heat release from freezing. A map of differences between mean $T_{\mathrm{DD}}$ at SD and LD onset (Fig. 4) does not reveal a strong regional signal over the NEUS/SECA. The largest $T_{\mathrm{DD}}$ values are slightly more common at LD onset than at SD onset in the regionally aggregated distributions (Fig. 5a), with a mean (median) of $2.2^{\circ} \mathrm{C}\left(2.0^{\circ} \mathrm{C}\right)$ at $\mathrm{LD}$ onset and $2.0^{\circ} \mathrm{C}\left(1.7^{\circ} \mathrm{C}\right)$ at $\mathrm{SD}$ onset $(p<0.001)$.

We now compare soundings taken at the onset of SD and LD events to examine differences in the 
a) NEUS/SECA

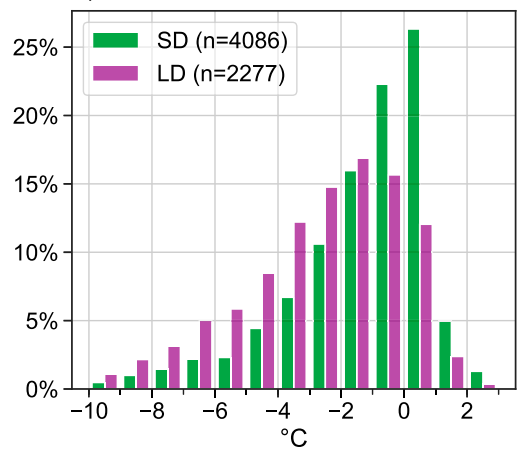

b) ScUS

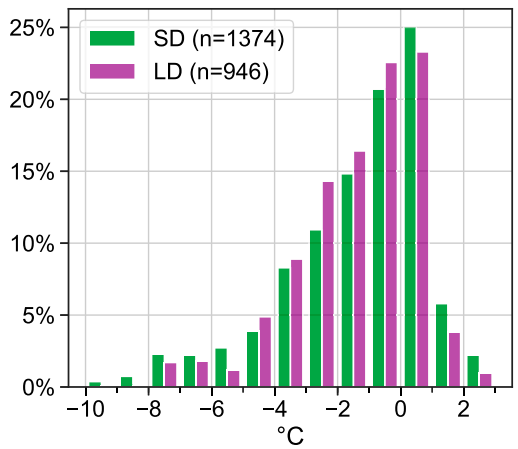

c) SEUS

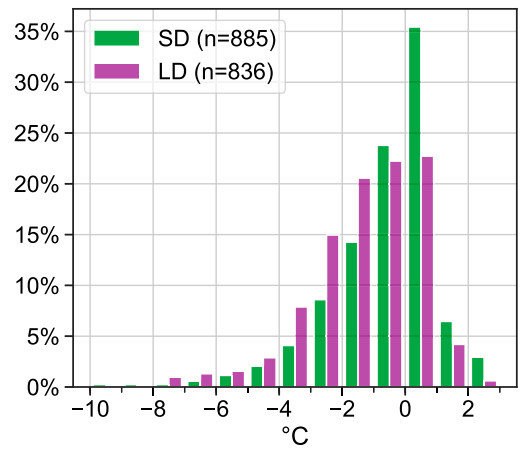

FIG. 3. Distributions of SD (green; left bar) and LD (purple; right bar) onset surface temperatures $\left({ }^{\circ} \mathrm{C}\right)$ in (a) the NEUS/SECA, (b) the SCUS, and (c) the SEUS. The bars plotted between $0^{\circ}$ and $1^{\circ} \mathrm{C}$, for example, represent the frequency of $0^{\circ} \mathrm{C} \leq T<1^{\circ} \mathrm{C}$.

thermodynamic profiles aloft. Figure 6a demonstrates that the surface station findings discussed above extend through the near-surface cold layer, with substantially colder temperature profiles at LD onset than at SD onset from the surface to $\approx 1000-1500 \mathrm{~m}$ AGL. The cold layer is deeper and colder at LD onset than at SD onset (Figs. 7a,b), with a median depth of $1012 \mathrm{~m}$ and $T_{\min }$ of $-4.8^{\circ} \mathrm{C}$ at LD onset compared with $596 \mathrm{~m}$ and $-2.6^{\circ} \mathrm{C}$ at SD onset ( $p=0.002$ for depth, $p<0.001$ for $\left.T_{\min }\right)$.

Conversely, the median profiles above the cold layer overlap considerably (Fig. 6a), and distributions of warm layer and cloud characteristics do not exhibit large differences between SD and LD events (Figs. $7 \mathrm{~d}-\mathrm{f}$ and $8, p>0.20$ for each of these fields). The $T_{\mathrm{DD}}$ profiles aloft (Fig. 6a) suggest near-saturated conditions throughout the lower troposphere at the onset of both event types, with very little spread, especially when compared with the SCUS or SEUS (Figs. 6b,c). Moisture availability does not appear to be a limiting factor for most freezing rain events in the NEUS/SECA. This is the region in which freezing rain events are least frequently followed by no precipitation or freezing drizzle (Table 1).

While differences in onset temperature and moisture profiles in the warm layer are minimal, temperature and moisture advection in the warm layer tend to be stronger at LD onset than at SD onset (Figs. 9b,d). Both categories largely begin with WAA $\left[-\mathbf{v} \cdot \nabla T>0^{\circ} \mathrm{C}(3 \mathrm{~h})^{-1}\right]$ at the level of $T_{\max }^{250-2500 \mathrm{~m}}(93 \%$ of $\mathrm{LD}$ events and $78 \%$ of SD events), with median values of $+3.0^{\circ} \mathrm{C}(3 \mathrm{~h})^{-1}$ at $\mathrm{LD}$ onset and $+2.1^{\circ} \mathrm{C}(3 \mathrm{~h})^{-1}$ at $\mathrm{SD}$ onset $(p=0.011$, Fig. 9b). Similarly, large percentages of both SD and LD events begin with positive moisture advection $\left[-\mathbf{v} \cdot \nabla w>0 \mathrm{~g} \mathrm{~kg}^{-1}(3 \mathrm{~h})^{-1}\right]$ at this level (98\% of LD events and $79 \%$ of SD events), but the magnitude is typically larger for LD events than for SD events $(p=0.006$, Fig. 9d). Surface temperature and moisture advection distributions are similar and straddle zero at both SD and LD onset (Figs. 9a,c). Strong surface CAA that could replenish subfreezing air is thus not systematically present for either event type.

As with the other previously described characteristics, SD and LD onset median wind profiles are almost identical above $\approx 2000 \mathrm{~m}$ AGL but vary substantially below this level (Fig. 6a). Consistent with the stronger WAA in LD events, the LD wind profiles veer more strongly, from southeasterly just above to surface to southwesterly near the top of the warm layer. Smallermagnitude veering is also evident in the median SD wind profile, from south-southeasterly to southwesterly over the same depth.

Approximately equal proportions of LD (52\%) and SD $(48 \%)$ events end with surface temperatures $\geq 0{ }^{\circ} \mathrm{C}$, resulting in a transition to rain in $43 \%$ of LD and $39 \%$ of SD events (Table 1). This propensity for both event types to end once surface temperatures reach $0^{\circ} \mathrm{C}$ highlights the importance of cold onset surface temperatures and cold layers in this region. The colder the surface temperature, the longer it will take for the

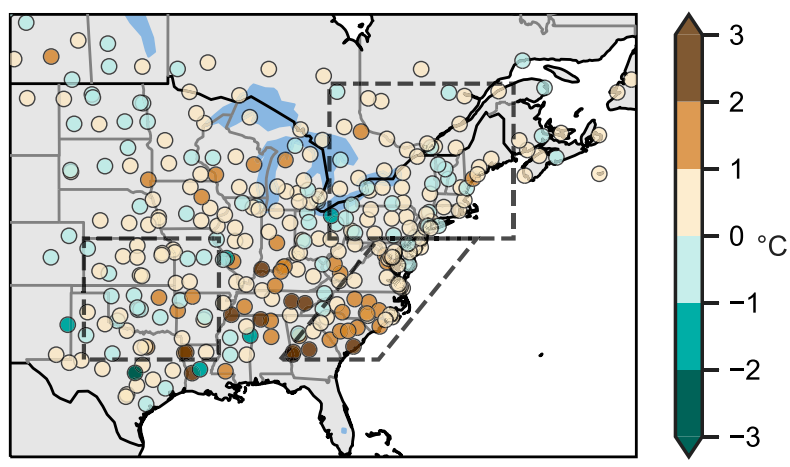

FIG. 4. As in Fig. 2, but showing the mean dewpoint depression observed at LD event onset minus the mean dewpoint depression observed at SD event onset $\left({ }^{\circ} \mathrm{C}\right)$ at each station. 
a) NEUS/SECA

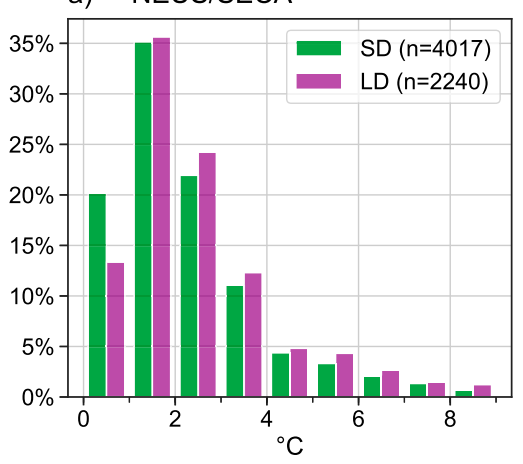

b) scus

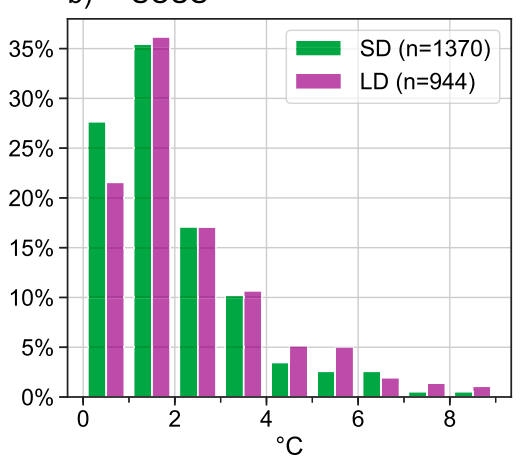

c) SEUS

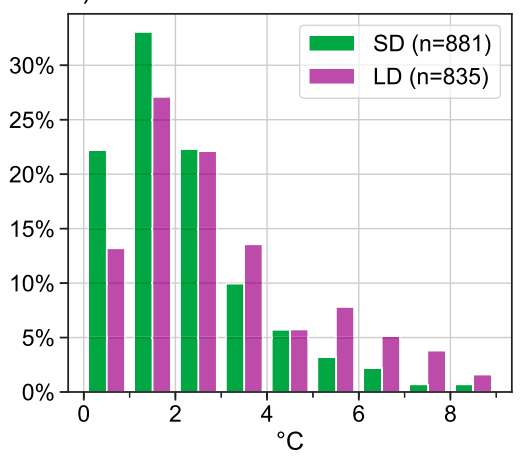

FIG. 5. Histograms as in Fig. 3, but for SD (green) and LD (purple) onset dewpoint depressions $\left({ }^{\circ} \mathrm{C}\right)$.

surface to reach $0^{\circ} \mathrm{C}$ for a given heating rate (e.g., via latent heat release or WAA).

\section{b. SCUS}

In the SCUS, McCray et al. (2019) found that LD events typically end as the warm layer cools and the atmospheric column dries aloft. This results in a transition from freezing rain to either no precipitation (32\% of both SD and LD events), freezing drizzle (31\% of LD and $22 \%$ of SD events), or snow/ice pellets ( $25 \%$ of LD and $35 \%$ of SD events) (Table 1). We also found surface CAA to usually be sufficient to maintain the cold layer throughout LD events, with surface temperatures remaining steady or decreasing slightly during LD events despite the latent heat released during freezing. These results suggest several precursors potentially distinguishing LD and SD events in the SCUS. First, particularly warm onset warm layers, or stronger WAA into the warm layer, may allow cooling mechanisms such as melting to persist for a longer period before erosion of the layer and an eventual transition to frozen precipitation. Additionally, a moister onset warm layer, or stronger moisture advection into the warm layer, could delay the transition to freezing drizzle or the cessation of precipitation.

Consistent with the suggestion that surface CAA is sufficient to offset any warming, onset surface temperature differences between SD and LD events do not show a coherent regional signal in the SCUS, with the mean LD onset temperature slightly warmer than the mean SD onset temperature at some stations (Fig. 2). Regionally, onset surface temperature distributions largely overlap, with identical mean (median) values $\left[-1.7^{\circ} \mathrm{C}\left(-1.0^{\circ} \mathrm{C}\right)\right](p=0.079$, Fig. 3b). Similarly, LD events are not associated with especially dry surface conditions at onset, though the distribution of surface $T_{\mathrm{DD}}$ does tend toward slightly larger values at $\mathrm{LD}$ onset than at SD onset (Fig. 5b).
As with the surface conditions, characteristics of the onset cold layer do not exhibit large differences between SD and LD events, with overlapping median low-level temperature profiles (Fig. 6b). Distributions of cold-layer $T_{\min }$ and depth are also similar for the two event categories (Figs. 7a,b, $p>0.20$ for both). The cold- and dry-air advection observed at the onset of most SD and LD events (Figs. 9a,c) appears to be sufficient to sustain the cold layer here, even with onset temperatures near $0^{\circ} \mathrm{C}$.

The profile above the cold layer, however, is substantially warmer at LD onset than at SD onset (Fig. 6b). Warm layers are deeper at LD onset than at SD onset (median depths of 1793 and $1095 \mathrm{~m}$, respectively) ( $p=$ 0.001 , Fig. $7 d)$. LD onset soundings also have a warmer median $T_{\max }^{250-2500 \mathrm{~m}}\left(5.1^{\circ} \mathrm{C}\right)$ compared with $2.7^{\circ} \mathrm{C}$ at $\mathrm{SD}$ onset ( $p=0.001$, Fig. 7f). A substantial proportion $(25 \%)$ of SD onset soundings lack a warm layer, compared with only $4 \%$ of LD onset soundings. Warm-layer $T_{\max }$ cannot be calculated for these soundings. Thus, the sample size is smaller and the $p$ value is larger for warmlayer $T_{\max }$ than for $T_{\max }^{250-2500 \mathrm{~m}}$ (Figs. 7e,f).

To explain the more frequent absence of a warm layer at SD onset compared with LD onset, we analyzed the individual thermodynamic profiles for each of the $16 \mathrm{SD}$ onset soundings lacking a warm layer as well as surface observations surrounding event onset (not shown). Eleven $(69 \%)$ of these cases exhibited thermodynamic profiles likely conducive to the supercooled warm rain process, with a saturated layer near the surface and cloud-top temperatures potentially too warm for ice nucleation $\left(\gtrsim-10^{\circ} \mathrm{C}\right)$. Rauber et al. (2000) found this process to be at least partly responsible for $47 \%$ of the freezing precipitation observations they examined, including several instances of freezing rain.

Additionally, the radiosonde may have been measuring the post-freezing rain environment. Freezing rain in this region is often associated with a cold frontal 


\section{a) NEUS/SECA}

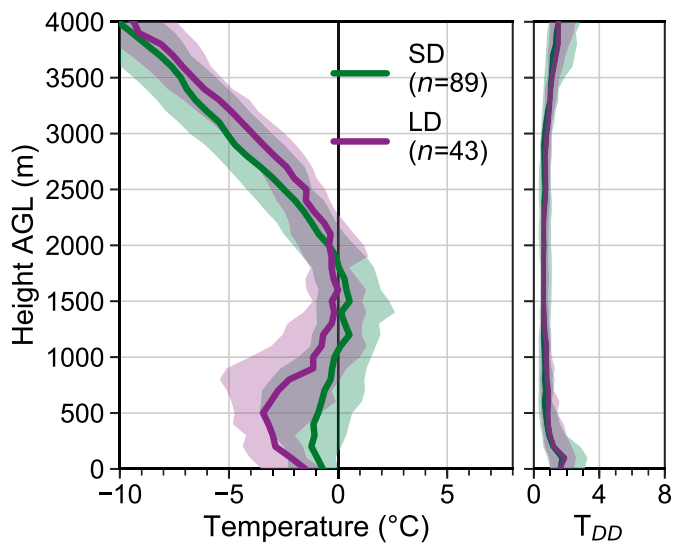

b) SCUS

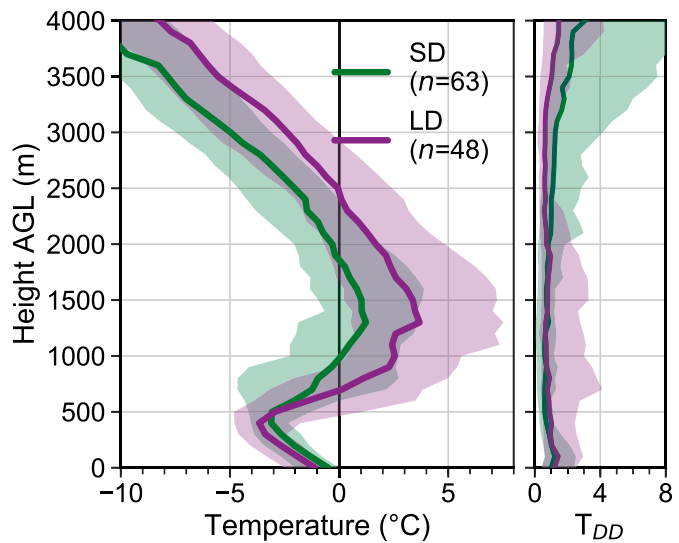

c) SEUS

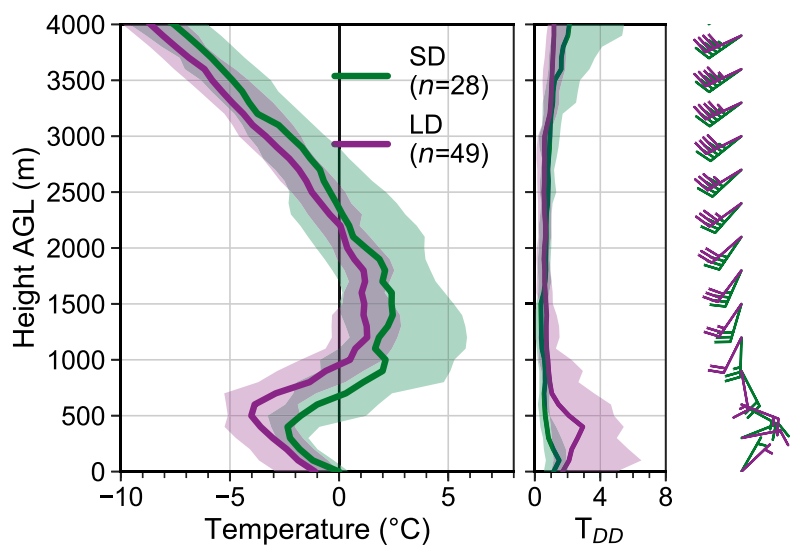

FIG. 6. Composites of observed soundings among all upper-air sites in (a) the NEUS/SECA, (b) the SCUS, and (c) the SEUS, showing (left) the median (solid lines) and interquartile range (shaded) of temperature $\left({ }^{\circ} \mathrm{C}\right.$ ) at each level, (center) the median (solid lines) and interquartile range (shaded) of dewpoint depression $T_{\mathrm{DD}}\left({ }^{\circ} \mathrm{C}\right)$, and (right) median winds at each level [barbs; fulllength barb is $10 \mathrm{kt}\left(\approx 5.14 \mathrm{~m} \mathrm{~s}^{-1}\right)$ ] at SD event onset (green) and LD event onset (purple). passage (e.g., Sanders et al. 2013; Mullens et al. 2016b), and SD events are commonly followed by snow/ice pellets $(35 \%)$ or freezing drizzle $(22 \%)$, neither of which require a $>0^{\circ} \mathrm{C}$ warm layer aloft (Table 1 ). In 15 of the 16 cases, surface temperatures decreased during the six hours preceding event onset. A rapid cold frontal passage would make the resulting sounding highly sensitive to the precise time of radiosonde release. We cannot be certain of the precipitation type occurring at the exact time of radiosonde measurement. Importantly, despite quantitative differences, qualitative results using only the cases with a warm layer are the same as those found when the soundings lacking a warm layer are included (cf. Figs. 7e,f). Warm-layer characteristics are therefore robust features distinguishing LD events from SD events in the SCUS.

In addition to temperature profile differences, conditions aloft tend to be drier at SD onset than at LD onset, with larger $T_{\mathrm{DD}}$ values above $\approx 2000 \mathrm{~m}$ AGL for SD events than LD events (Fig. 6b). Cloud layers tend to be deeper at LD onset (median $4906 \mathrm{~m}$ ) than at SD onset (median $3670 \mathrm{~m})(p=0.022$, Fig. 8a). Consequently, though distributions are more similar than for depth, cloud-top temperature distributions are colder at $\mathrm{LD}$ onset (median $-16.8^{\circ} \mathrm{C}$ ) than at SD onset $\left(\right.$ median $\left.-10.8^{\circ} \mathrm{C}\right)(p=0.091$, Fig. $8 b)$.

Temperature and moisture advection in the warm layer are also stronger (more positive) at LD onset than at SD onset, with $p<0.001$ for comparisons of SD and LD distributions of both characteristics. Median temperature advection at the level of $T_{\max }^{250-2500 \mathrm{~m}}$ is $1.8^{\circ} \mathrm{C}$ $(3 \mathrm{~h})^{-1}$ at $\mathrm{LD}$ onset and $0.5^{\circ} \mathrm{C}(3 \mathrm{~h})^{-1}$ at SD onset (Fig. $9 \mathrm{~b}$ ), with CAA observed at this level at the onset of only $15 \%$ of LD events but $43 \%$ of SD events. Median moisture advection at this level is $0.8 \mathrm{~g} \mathrm{~kg}^{-1}(3 \mathrm{~h})^{-1}$ at LD onset compared with $0.1 \mathrm{~g} \mathrm{~kg}^{-1}(3 \mathrm{~h})^{-1}$ at SD onset, with dryair advection $\left[-\mathbf{v} \cdot \nabla w<0 \mathrm{~g} \mathrm{~kg}^{-1}(3 \mathrm{~h})^{-1}\right]$ at this level less common at the onset of LD events $(17 \%)$ than SD events $(41 \%)$.

Differences in warm-layer onset temperature and moisture profiles and their advections are consistent with the differences in median wind profiles between SD and LD events in the SCUS (Fig. 6b). While the median winds in the LD warm layer veer from southsoutheasterly to southwesterly with height, winds in the SD warm layer lack strong veering and are primarily west-southwesterly. These differences suggest variations in the source region of air parcels entering the warm layer between the event types. The southsoutheasterly flow at the bottom of the LD warm layer suggests parcel trajectories originating over the Gulf of Mexico, as identified in past studies of ice storms in this region (Sanders et al. 2013; Mullens et al. 2016a,b). 
a) Cold layer depth $(\mathrm{m})$

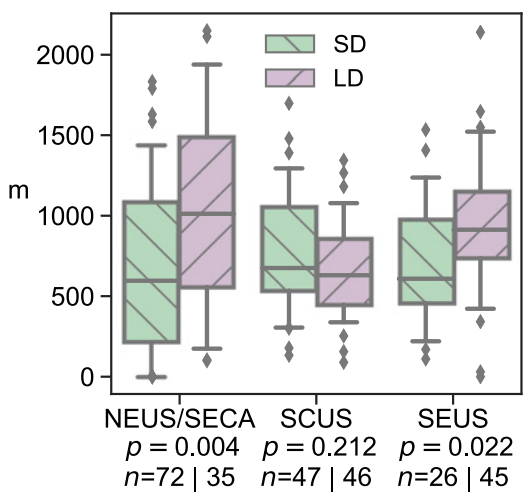

d) Warm layer depth (m)

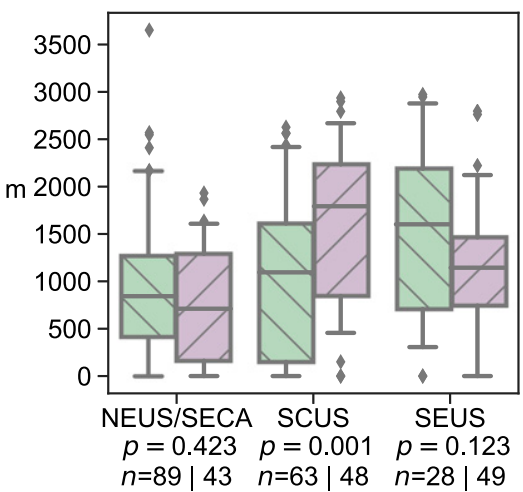

b) Cold layer $\mathrm{T}_{\min }\left({ }^{\circ} \mathrm{C}\right)$

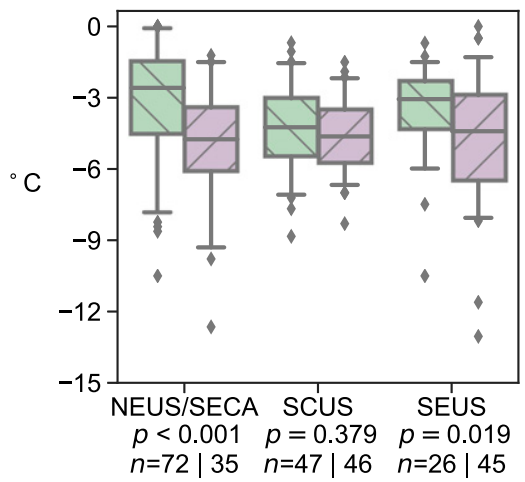

e) Warm layer $T_{\max }\left({ }^{\circ} \mathrm{C}\right)$

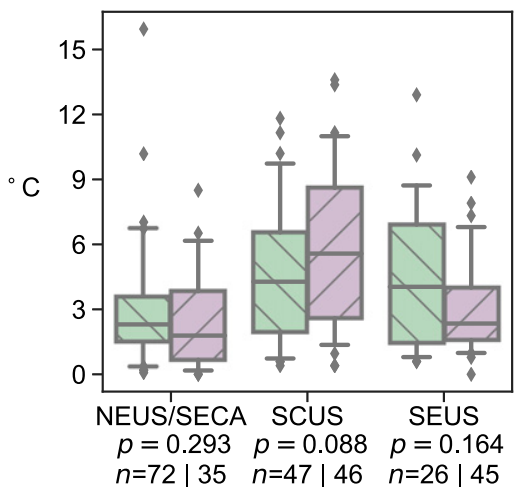

c) $T_{\min } 0-1000 \mathrm{~m} \mathrm{AGL}\left({ }^{\circ} \mathrm{C}\right)$

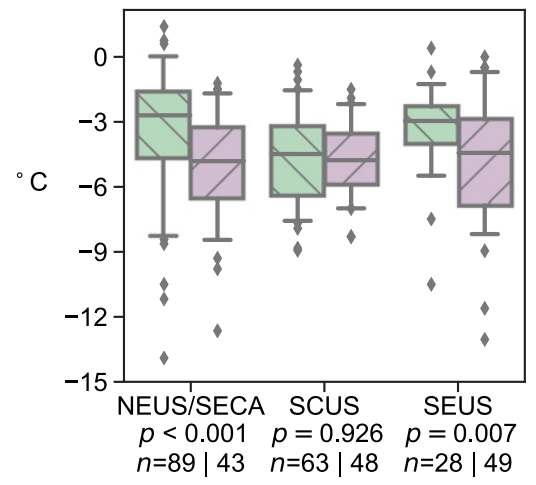

f) $T_{\text {max }} 250-2500 \mathrm{~m} \mathrm{AGL}\left({ }^{\circ} \mathrm{C}\right)$

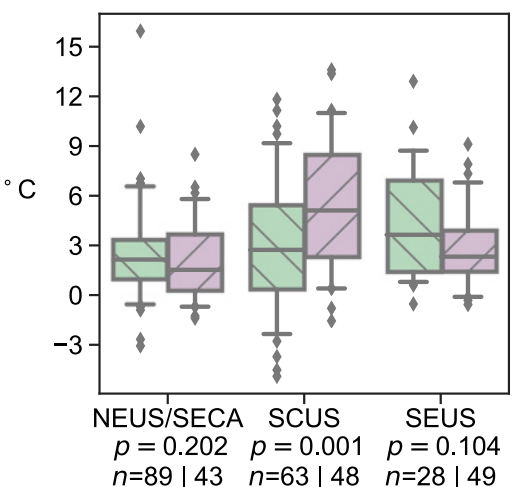

FIG. 7. Boxplots displaying distributions of (a) the depth and (b) the minimum temperature in the cold layer, (c) the minimum temperature in the 0-1000 m AGL layer, (d) the depth and (e) maximum temperature in the warm layer, and (f) the maximum temperature in the 250-2500 m AGL layer at SD event onset (left box; green) and LD event onset (right box; purple) for each focus region. Differences between the SD and LD distributions are evaluated using the two-sided Mann-Whitney $U$ test, with resulting $p$ values displayed below region names. Sample sizes for each event type and region are displayed as $n_{\mathrm{SD}} \mid n_{\mathrm{LD}}$. Boxplots display the interquartile range (shaded), the median (horizontal line), the 5th percentile and the 95th percentile (ends of lower and upper whiskers, respectively), and outliers beyond these percentile ranges (diamonds).

The southwesterly flow in the SD composite is instead potentially indicative of overland trajectories. This also implies distinctions in synoptic patterns leading to the two event types, particularly the warm-layer mass fields.

Mullens (2014) compared thermodynamic profiles observed within one hour of freezing rain observations during heavy [ $>0.25 \mathrm{in} .(6.35 \mathrm{~mm})$ liquid equivalent freezing rain accumulation] and light $(\leq 0.25 \mathrm{in}$.) freezing rain events in the southern Great Plains of the United States, similar to our SCUS region, from 1993 to 2011. These precipitation amounts were estimates based on available observations, as mixed precipitation during events complicates the partitioning of amounts by phase. Our results, using a larger sample (primarily owing to our longer study period), agree well with their findings that heavy freezing rain events were distinguished from light cases by their warmer, deeper warm layers. For example, $92 \%$ of the heavy freezing rain soundings they examined had a maximum warm-layer wet-bulb temperature $>5^{\circ} \mathrm{C}$, compared with $27 \%$ of light freezing rain soundings. Mullens (2014) also found that cloud layers were $>2.5 \mathrm{~km}$ deep in $60 \%$ of heavy freezing rain soundings but only $33 \%$ of light freezing rain soundings, consistent with our results.

Several key mechanisms or onset characteristics can therefore support prolonged freezing rain in the SCUS. First, a warmer onset warm layer can undergo cooling due to melting or CAA for a longer period before it completely erodes and precipitation transitions to snow or ice pellets. Similarly, stronger WAA into the layer can offset some of this cooling and sustain freezing rain. Finally, more saturated conditions aloft at onset or advection of moist air aloft during events can maintain cloud layers and delay the cessation of precipitation or transition to freezing drizzle.

\section{c. SEUS}

In the SEUS, as in the NEUS/SECA, LD events most commonly end in a transition from freezing rain to 
a) Cloud depth (m)

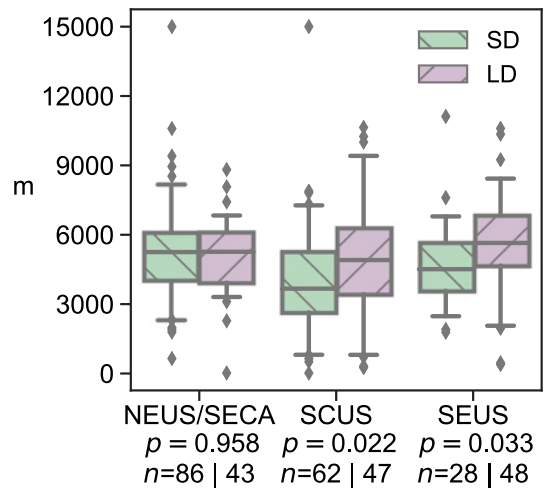

b) Cloud top temperature $\left({ }^{\circ} \mathrm{C}\right)$

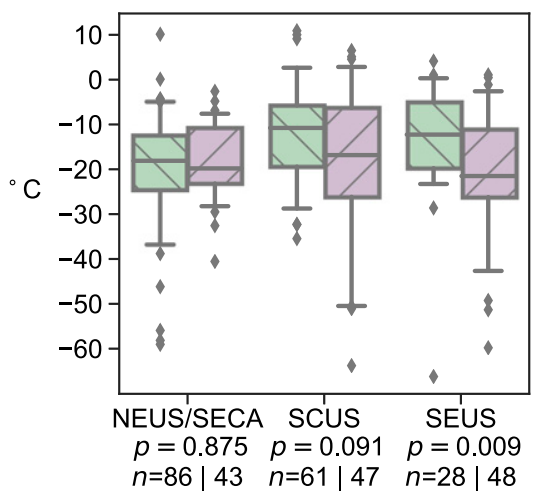

FIG. 8. Boxplots as in Fig. 7, but comparing the SD and LD onset distributions of (a) the depth of the cloud layer (m) and (b) the cloud-top temperature $\left({ }^{\circ} \mathrm{C}\right)$.

rain/drizzle as the cold layer erodes and the surface warms to $0^{\circ} \mathrm{C}$ (McCray et al. 2019). Additionally, onset $T_{\mathrm{DD}}$ values there are larger than in the other regions, and evaporative cooling of raindrops falling through the cold layer appears to partially offset low-level warming. These results suggest that colder, drier onset cold layers may lead to longer-duration events in this region.

First, colder 2-m temperatures are evident at LD event onset than at SD onset at most SEUS surface stations, though the magnitude of these differences is not as large as in the NEUS/SECA (Fig. 2). The regional mean (median) onset temperature is $-1.5^{\circ} \mathrm{C}\left(-1.1^{\circ} \mathrm{C}\right)$ for $\mathrm{LD}$ events compared with $-0.9^{\circ} \mathrm{C}\left(-0.6^{\circ} \mathrm{C}\right)$ for SD events $(p<0.001$, Fig. $3 \mathrm{c})$. A relatively high frequency of onset temperatures $\geq-1{ }^{\circ} \mathrm{C}$ for both event types (50\% of LD events and $69 \%$ of SD events) suggests that especially cold onset temperatures are less vital for persistent freezing rain in the SEUS than in the NEUS/SECA, where only $30 \%$ of LD events begin with $T \geq-1{ }^{\circ} \mathrm{C}$.

Evaporative cooling is a particularly important mechanism for cold-layer maintenance in the SEUS, with a coherent pattern of stations with mean $T_{\mathrm{DD}}$ values that are $\geq 1^{\circ} \mathrm{C}$ larger at LD onset than at SD onset (Fig. 4). Regionally, the mean (median) onset $T_{\mathrm{DD}}$ is $2.9^{\circ} \mathrm{C}$ $\left(2.2^{\circ} \mathrm{C}\right)$ for $\mathrm{LD}$ events and $2.0^{\circ} \mathrm{C}\left(1.7^{\circ} \mathrm{C}\right)$ for $\mathrm{SD}$ events $(p<0.001$, Fig. 5 c). $60 \%$ of $\mathrm{LD}$ events have onset $T_{\mathrm{DD}} \geq 2^{\circ} \mathrm{C}$ compared with $45 \%$ of SD events. This is consistent with the Bell and Bosart (1988) finding that evaporative cooling is a key mechanism for developing near-surface cold air during Appalachian cold-air damming (CAD), which is often associated with freezing rain in this region (e.g., Forbes et al. 1987; Bernstein 2000; Rauber et al. 2001; Robbins and Cortinas 2002).

In the median soundings, these differences extend through the cold layer, with the 75th percentile of $T_{\mathrm{DD}}$ reaching $\approx 6^{\circ} \mathrm{C}$ for LD events, but only $\approx 3^{\circ} \mathrm{C}$ for $\mathrm{SD}$ events (Fig. 6c). The median cold layer is deeper and colder for LD events than for SD events, with a median depth and $T_{\min }$ of $913 \mathrm{~m}$ and $-4.4^{\circ} \mathrm{C}$ at LD onset and $603 \mathrm{~m}$ and $-3.1^{\circ} \mathrm{C}$ at SD onset ( $p=0.022$ for depth and $p=0.019$ for $\left.T_{\min }\right)$ (Figs. 7a,b). Notably, the SD and LD profiles through the lowest $\approx 500 \mathrm{~m}$ of the cold layer overlap substantially, with the largest differences being in the upper portion of the layer. The greatest difference in cold layers between the two event types is therefore the depth, rather than $T_{\min }$.

Warm-layer characteristics in the SEUS exhibit larger spread at SD onset than at LD onset, with a tendency toward a slightly less prominent warm layer at LD onset (Figs. 7d-f) consistent with a colder/deeper cold layer. Though $p$ values for warm-layer characteristics are all $>0.10$, the LD distributions are primarily constrained to smaller depths/colder $T_{\max }$ values compared with SD distributions (Figs. $7 \mathrm{~d}-\mathrm{f}$ ). The median $T_{\max }$ is $2.3^{\circ} \mathrm{C}$ at LD onset and $4.0^{\circ} \mathrm{C}$ at SD onset $(p=0.164)$, with a median depth of $1144 \mathrm{~m}$ at LD onset and $1602 \mathrm{~m}$ at SD onset $(p=0.123)$.

LD events are also associated with colder cloud tops at onset than SD events, with a median cloud-top temperature of $-21.5^{\circ} \mathrm{C}$ at $\mathrm{LD}$ onset and $-12.3^{\circ} \mathrm{C}$ at SD onset ( $p=0.009$, Fig. $8 \mathrm{~b}$ ). Cloud layers also tend to be deeper at LD onset (median $5646 \mathrm{~m}$ ) than at SD onset (median $4511 \mathrm{~m})(p=0.033$, Fig. 8a). Warmer/shallower cloud layers at SD onset may be due to the radiosonde sampling some events as freezing rain is ending, as a combined $42 \%$ of SEUS SD events end in no precipitation or freezing drizzle (Table 1). In McCray et al. (2019), we found dry-air advection at the level of $T_{\max }^{250-2500 \mathrm{~m}} \mathrm{oc}-$ curring at the end of some LD events in this region. As in the SCUS, colder/deeper clouds at onset may therefore help sustain freezing rain for a longer period in the face of dry-air advection eroding the cloud layer.

As $54 \%$ of LD and $52 \%$ of SD events end with surface temperatures $\geq 0^{\circ} \mathrm{C}$, freezing rain most commonly 
TABLE 1. Percentage of LD (SD) freezing rain events followed and preceded by snow/ice pellets (SN/IP), rain/drizzle (RA/DZ), freezing drizzle (FZDZ), or no precipitation (NP) among all stations in the NEUS/SECA, SCUS, and SEUS regions. Bold text indicates the most common phase preceding or following each event type at each region and period. Sums of percentages may be $>100 \%$, as mixes can be counted for multiple categories. Only observations from 1979 to 1994 (roughly the preautomation era) are used. Sample sizes for LD (SD) events are $n=1219$ (2104) in the NEUS/SECA, $n=423$ (612) in the SCUS, and $n=451$ (460) in the SEUS. Table and caption modified from Table 1 in McCray et al. (2019) to include SD events.

\begin{tabular}{|c|c|c|c|c|c|c|}
\hline \multirow[b]{2}{*}{ Type } & \multicolumn{3}{|c|}{ Before event onset } & \multicolumn{3}{|c|}{ After event end } \\
\hline & NEUS/SECA & SCUS & SEUS & NEUS/SECA & SCUS & SEUS \\
\hline SN/IP & $51 \%(48 \%)$ & $16 \%(20 \%)$ & $43 \%(40 \%)$ & $23 \%(29 \%)$ & $25 \%(\mathbf{3 5} \%)$ & $14 \%(18 \%)$ \\
\hline $\mathrm{RA} / \mathrm{DZ}$ & $12 \%(19 \%)$ & $32 \%(30 \%)$ & $34 \%(38 \%)$ & $43 \%(39 \%)$ & $18 \%(14 \%)$ & $45 \%(43 \%)$ \\
\hline FZDZ & $12 \%(11 \%)$ & $15 \%(15 \%)$ & $7 \%(6 \%)$ & $16 \%(13 \%)$ & $31 \%(22 \%)$ & $21 \%(16 \%)$ \\
\hline NP & $31 \%(29 \%)$ & $43 \%(42 \%)$ & $26 \%(24 \%)$ & $22 \%(23 \%)$ & 32\% $(32 \%)$ & $24 \%(26 \%)$ \\
\hline
\end{tabular}

transitions to rain/drizzle for both categories (Table 1). Thus, a colder onset cold layer can sustain heating via freezing for a longer period before the surface reaches $0^{\circ} \mathrm{C}$. The role of evaporative cooling is especially important in the SEUS compared with the other two regions, as particularly dry onset cold layers allow evaporative cooling to offset heating via WAA aloft and freezing at the surface for a longer period than if the near-surface environment were more saturated.

\section{Discussion}

Several studies have explored sounding characteristics associated with North American freezing rain events (Czys et al. 1996; Zerr 1997; Rauber et al. 2001; Robbins and Cortinas 2002). For example, Zerr (1997) examined 34 soundings observed during freezing rain, freezing drizzle, and ice pellets at stations in the United States and southern Canada, but did not perform any regional comparisons. Rauber et al. (2001) grouped soundings into seven categories based on the synoptic pattern responsible for freezing precipitation. Robbins and Cortinas (2002) compared soundings observed during freezing rain at six individual sounding sites in the United States, noting large regional differences in the characteristics of the warm and cold layers.

Our study expands on past results in two key aspects. First, past studies have not compared freezing rain events based on their duration. To our knowledge, only Mullens (2014) has identified differences between weak and severe freezing rain events, and did so only for the southern Great Plains of the United States. Our results for the similar SCUS region using an expanded sample [63 SD and 48 LD events, compared with 53 light and 25 heavy events for Mullens (2014)] and differing methodology (duration rather than precipitation amount) agree well with theirs. In addition, our analysis expands to the NEUS/SECA and SEUS.
Additionally, past studies of soundings during freezing rain have generally used any sounding taken during freezing rain, regardless of at what point during the event the sounding was observed. This is largely because the rarity of freezing rain events and the twice-daily nature of radiosonde observations have complicated attempts to develop a sufficiently large sample for robust statistical analysis. Because thermodynamic profiles evolve throughout events (e.g., McCray et al. 2019), statistics calculated from these profiles are sensitive to when in the event life cycle the sounding was observed. Our technique of grouping soundings regionally has allowed us to examine characteristics specifically at event onset while maintaining a reasonably large sample for each region. This focus on onset may facilitate the use of these results for forecast applications, as forecasters can compare our findings to predicted thermodynamic profiles at the time freezing rain is expected to begin.

The large differences in sounding characteristics even among proximate stations examined by Robbins and Cortinas (2002) complicate the use of threshold values of warm/cold-layer characteristics for forecasting precipitation type. In particular, they noted that the median cold-layer depth of $1000 \mathrm{~m}$ at Albany, New York (KALB), was nearly twice that at Buffalo, New York (KBUF, $503 \mathrm{~m}$ ). They hypothesized that the position of Albany in the Hudson Valley allowed for cold air to build and persist for longer than at Buffalo, which lacks similar terrain features. We propose that terrain features supporting the characteristics identified here, namely, a deeper/colder cold layer in the NEUS/SECA, play a key role in enhancing the frequency of freezing rain at individual locations. For example, from 1979 to 2016, Albany observed $82 \%$ more LD freezing rain events (67) than Buffalo (37). Similarly, $33 \%$ of freezing rain events at Albany are long duration, compared with 23\% at Buffalo. Additional studies using numerical simulations to isolate the impacts of local terrain features could provide further insight. 

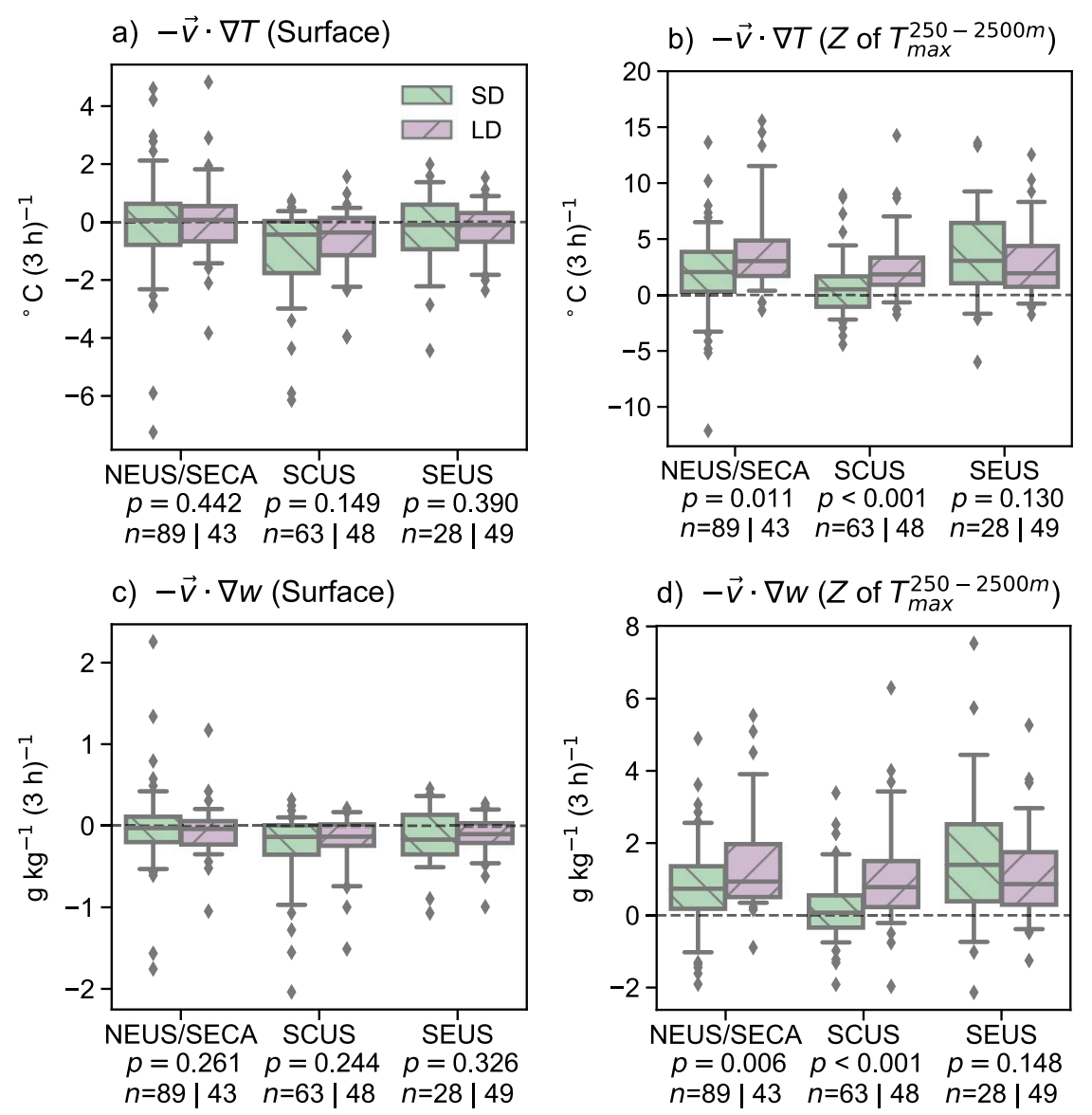

FIG. 9. Boxplots as in Fig. 7, but for distributions of (a),(b) temperature advection $[-\mathbf{v} \cdot \nabla T$; $\left.{ }^{\circ} \mathrm{C}(3 \mathrm{~h})^{-1}\right]$ and (c),(d) water vapor mixing ratio advection $\left[-\mathbf{v} \cdot \nabla w ; \mathrm{g} \mathrm{kg}^{-1}(3 \mathrm{~h})^{-1}\right]$ at (left) the surface and (right) at the altitude of $T_{\max }^{250-2500 \mathrm{~m}}$.

Though we have identified salient thermodynamic features distinguishing SD from LD events in each region, factors such as the aforementioned localized terrain features must be considered when applying our findings to a given region. For example, though surface temperature advection is generally weak at both SD and LD onset in the NEUS/SECA, surface CAA may be an important mechanism in valleys that support channeling of near-surface cold air toward particular stations during events (e.g., Razy et al. 2012; Ressler et al. 2012), replenishing the cold layer and offsetting heating due to WAA or latent heat release. Studies focusing on local differences between short- and long-duration freezing rain events in these regions could provide additional useful information for local forecasters.

An additional factor impacting surface heating during freezing rain events that has not been explored here is land surface conditions. Lackmann et al. (2002) noted that for bare ground, the proportion of the latent heat released upon freezing that will be transferred into the ground compared with into the air increases for colder soil temperatures. When the ground is warm or insulated by snow, more of the latent heat released acts to warm the air. Lackmann et al. (2002) identified the fraction $F_{A}$ of latent heat that warms the atmosphere as a scale factor for their Eq. (5), which estimates ice accretion given initial cold-layer conditions. Additional observational or modeling studies that examine the effect of soil conditions on freezing rain event duration and help constrain values of $F_{A}$ could expand on the results found here.

Finally, the differences we have identified in the wind profiles and magnitudes of temperature/moisture advection aloft (Figs. 6 and 9) also suggest the synopticdynamic patterns leading to SD and LD events may vary substantially. Future work comparing these patterns could help elucidate the key features necessary for prolonged freezing rain in each region. 


\section{Summary}

In this paper, we have identified salient thermodynamic features that commonly differentiate long-duration freezing rain events from short-duration cases that are less likely to result in severe damage. These key differences vary greatly between regions.

In the NEUS/SECA, both SD and LD freezing rain events are typically preceded by frozen precipitation as vertical temperature profiles are entirely $<0^{\circ} \mathrm{C}$. Precipitation transitions to freezing rain as strong WAA aloft develops the warm layer. This evolution is consistent with studies of freezing rain at various locations in the NEUS/SECA that identified approaching warm fronts as the primary mechanism for freezing rain production there (e.g., Cortinas 2000; Rauber et al. 2001; Robbins and Cortinas 2002; Ressler et al. 2012; Castellano 2012).

Large-scale lower-tropospheric CAA is not typically observed during freezing rain events in the NEUS/SECA. Lacking this replenishment of cold air, the release of latent heat of fusion when rain freezes at the surface and WAA aloft act to erode the cold layer during events. Colder surface temperatures and colder/deeper cold layers at event onset are associated with longer-duration events, as the aforementioned heating mechanisms can occur over a longer period before the surface reaches $0^{\circ} \mathrm{C}$ and precipitation transitions to rain. For a given heating rate, the surface will simply reach $0^{\circ} \mathrm{C}$ more quickly if the temperature is warmer when freezing rain begins.

In agreement with the findings of Mullens (2014) comparing heavy and light freezing rain events, and in contrast with the other two regions studied, we have found that longer-duration freezing rain events in the SCUS occur under generally warmer lower-tropospheric onset conditions. Past studies have shown freezing rain events in this region to begin following the passage of a cold front at the leading edge of a southwardpropagating arctic anticyclone (Sanders et al. 2013; Mullens et al. 2016b). Accordingly, surface CAA is common during both SD and LD events, and surface temperatures do not tend to warm substantially during events. LD events in the SCUS end as the warm layer erodes and profiles dry, causing freezing rain to either end or transition to frozen precipitation or freezing drizzle (McCray et al. 2019). SD events follow a similar evolution, with slightly more frequent transitions to frozen precipitation than for LD events (Table 1).

Unlike in the NEUS/SECA, the warm layer, rather than the cold layer, is the crucial factor in freezing rain event persistence in the SCUS. The extraction of latent heat of fusion when snowflakes melt as they fall through the warm layer can cool and erode the layer. The deeper, warmer warm layer found at LD event onset than at SD onset can sustain this cooling for a longer period, postponing warm-layer erosion to the point that complete melting no longer occurs and freezing rain changes to ice pellets or snow. Additionally, the warm layer is replenished by stronger WAA at LD onset than at SD onset. The CAA into the warm layer present at the onset of many SD cases accelerates warm-layer erosion and freezing rain cessation in those cases.

Upper-level moisture characteristics at onset also differ between SD and LD events in the SCUS. LD events are associated with both deeper cloud layers and stronger moisture advection into the warm layer at onset than SD events. SD events are characterized by shallower cloud layers and, in some cases, dry-air advection into the warm layer. In addition to preventing freezing rain from ending or transitioning to freezing drizzle, the more-saturated profile near the top of the warm layer evident in the LD onset composite may also limit potential cooling from sublimation of snowflakes or evaporation of raindrops, which could further erode the warm layer from above.

Finally, freezing rain in the SEUS is typically associated with Appalachian CAD (Bernstein 2000; Rauber et al. 2001; Robbins and Cortinas 2002). Wind and thermodynamic profiles examined here for both SD and LD events closely resemble profiles found in past studies of CAD in this region (Forbes et al. 1987; Bell and Bosart 1988), with north-northeasterly surface winds becoming easterly just above the surface and veering to southerly above the CAD cold dome.

In McCray et al. (2019), we found a similar event evolution in the SEUS as in the NEUS/SECA, with a transition from frozen precipitation to freezing rain to rain being most common. Accordingly, as in the NEUS/SECA, surface and cold-layer temperatures are colder, and the cold layer deeper, at LD onset than at SD onset, allowing heating via freezing at the surface and WAA aloft to persist for longer before the cold layer erodes.

Dry surface air at freezing rain onset is especially important for event persistence in the SEUS. LD events begin with larger $T_{\mathrm{DD}}$ values at the surface and through the cold layer than SD events. Raindrops falling into the subsaturated cold layer will begin to evaporate, with the resultant evaporative cooling partially offsetting the warming from WAA near the top of the cold layer and from the release of latent heat of fusion when rain freezes at the surface. When the surface is drier, this evaporative cooling can occur for a longer period before the air reaches saturation. The near-saturated cold layer commonly observed at SD onset reduces the potential 
for evaporation and its associated cooling, and thus heating mechanisms can quickly erode the cold layer and cause a rapid transition to rain.

The distinguishing characteristics identified here may be useful to forecasters in each of these regions, suggesting particular metrics on which to focus when attempting to predict the eventual duration of a freezing rain event. In the NEUS/SECA, particular attention should be placed on identifying whether particularly cold (i.e., several degrees $<0^{\circ} \mathrm{C}$ ) surface and cold-layer temperatures will be present when freezing rain is expected to begin. Similarly, onset surface and cold-layer temperatures should be examined closely in the SEUS. There, however, special attention should be placed on the moisture characteristics at the surface. If the surface $T_{\mathrm{DD}}$ at onset is small $\left(\lesssim 2^{\circ} \mathrm{C}\right)$, either an especially cold onset cold layer or stronger-than-usual surface CAA may be necessary to sustain the cold layer, and thus freezing rain, for a prolonged period. In the SCUS, freezing rain almost always occurs with surface CAA, and thus less focus on onset surface temperature is necessary. Instead, forecasters should closely examine conditions impacting the $>0{ }^{\circ} \mathrm{C}$ warm layer, including both onset conditions and the wind and associated temperature/moisture advection profiles in the layer. Future work identifying thresholds in these characteristics that may signify a much greater likelihood of a prolonged freezing rain event could allow for the development of forecast tools to aid in the prediction of these especially hazardous events.

Acknowledgments. This work has been supported by the Fonds de recherche du Québec-Nature et technologies (FRQNT) through the first author's doctoral research scholarship as well as a team grant, and by the Natural Sciences and Engineering Research Council of Canada (NSERC), through their Discovery Grant program and via the Canadian Network for Regional Climate and Weather Processes (CNRCWP). We thank Esther Mullens, Gary Lackmann, and two anonymous reviewers for their comments that led to substantial improvements to this manuscript.

\section{REFERENCES}

Bell, G. D., and L. F. Bosart, 1988: Appalachian cold-air damming. Mon. Wea. Rev., 116, 137-161, https://doi.org/10.1175/ 1520-0493(1988)116<0137:ACAD>2.0.CO;2.

Bernstein, B. C., 2000: Regional and local influences on freezing drizzle, freezing rain, and ice pellet events. Wea. Forecasting, 15, 485-508, https://doi.org/10.1175/1520-0434(2000)015<0485: RALIOF $>2.0 . \mathrm{CO} ; 2$.

Bocchieri, J. R., 1980: The objective use of upper air soundings to specify precipitation type. Mon. Wea. Rev., 108, 596-603, https:// doi.org/10.1175/1520-0493(1980)108<0596:TOUOUA > 2.0.CO;2.
Brooks, C. F., 1920: The nature of sleet and how it is formed. Mon. Wea. Rev., 48, 69-72, https://doi.org/10.1175/1520-0493(1920) $48<69$ b:TNOSAH $>2.0$.CO;2.

Castellano, C. M., 2012: Synoptic and mesoscale aspects of ice storms in the northeastern U.S. M.S. thesis, Dept. of Atmospheric and Environmental Sciences, University at Albany, State University of New York, 149 pp.

Changnon, S. A., 2003: Characteristics of ice storms in the United States. J. Appl. Meteor., 42, 630-639, https://doi.org/10.1175/ 1520-0450(2003)042<0630:COISIT > 2.0.CO;2.

Cortinas, J., 2000: A climatology of freezing rain in the Great Lakes region of North America. Mon. Wea. Rev., 128, 3574-3588, https:// doi.org/10.1175/1520-0493(2001)129<3574:ACOFRI>2.0.CO;2.

—_, B. C. Bernstein, C. C. Robbins, and J. W. Strapp, 2004: An analysis of freezing rain, freezing drizzle, and ice pellets across the United States and Canada: 1976-90. Wea. Forecasting, 19, 377-390, https://doi.org/10.1175/ 1520-0434(2004)019<0377:AAOFRF>2.0.CO;2.

Czys, R. R., R. W. Scott, K. C. Tang, R. W. Przybylinski, and M. E. Sabones, 1996: A physically based, nondimensional parameter for discriminating between locations of freezing rain and ice pellets. Wea. Forecasting, 11, 591-598, https://doi.org/10.1175/ 1520-0434(1996)011<0591:APBNPF>2.0.CO;2.

DeGaetano, A. T., 2000: Climatic perspective and impacts of the 1998 northern New York and New England ice storm. Bull. Amer. Meteor. Soc., 81, 237-254, https://doi.org/10.1175/ 1520-0477(2000)081<0237:CPAIOT > 2.3.CO;2.

Forbes, G. S., D. W. Thomson, and R. A. Anthes, 1987: Synoptic and mesoscale aspects of an Appalachian ice storm associated with cold-air damming. Mon. Wea. Rev., 115, 564-591, https:// doi.org/10.1175/1520-0493(1987)115<0564:SAMAOA>2.0.CO;2.

Gyakum, J. R., and P. J. Roebber, 2001: The 1998 ice storm-Analysis of a planetary-scale event. Mon. Wea. Rev., 129, 2983-2997, https:// doi.org/10.1175/1520-0493(2001)129<2983:TISAOA>2.0.CO;2.

Henson, W., R. Stewart, and B. Kochtubajda, 2007: On the precipitation and related features of the 1998 ice storm in the Montréal area. Atmos. Res., 83, 36-54, https://doi.org/10.1016/ j.atmosres.2006.03.006.

Huffman, G. J., and G. A. Norman, 1988: The supercooled warm rain process and the specification of freezing precipitation. Mon. Wea. Rev., 116, 2172-2182, https://doi.org/10.1175/ 1520-0493(1988)116<2172:TSWRPA > 2.0.CO;2.

Hurlbert, S. H., R. A. Levine, and J. Utts, 2019: Coup de grâce for a tough old bull: "Statistically significant" expires. Amer. Stat., $\mathbf{7 3}$ (Suppl. 1), 352-357, https://doi.org/10.1080/00031305.2018.1543616.

Kain, J. S., S. M. Goss, and M. E. Baldwin, 2000: The melting effect as a factor in precipitation-type forecasting. Wea. Forecasting, 15, 700-714, https://doi.org/10.1175/1520-0434(2000)015<0700: TMEAAF $>2.0 . \mathrm{CO} ; 2$.

Lackmann, G. M., 2011: Midlatitude Synoptic Meteorology: Dynamics, Analysis and Forecasting. Amer. Meteor. Soc., 345 pp.

— , K. Keeter, L. G. Lee, and M. B. Ek, 2002: Model representation of freezing and melting precipitation: Implications for winter weather forecasting. Wea. Forecasting, 17, 1016-1033, https:// doi.org/10.1175/1520-0434(2003)017<1016:MROFAM > 2.0.CO;2.

McCray, C. D., E. H. Atallah, and J. R. Gyakum, 2019: Longduration freezing rain events over North America: Regional climatology and thermodynamic evolution. Wea. Forecasting, 34, 665-681, https://doi.org/10.1175/WAF-D-18-0154.1.

Meisinger, C. L., 1920: The precipitation of sleet and the formation of glaze in the eastern United States, January 20 to 25, 1920, with remarks on forecasting. Mon. Wea. Rev., 48, 73-80, https:// doi.org/10.1175/1520-0493(1920)48<73b:TPOSAT>2.0.CO;2. 
Mullens, E. D., 2014: Moisture and thermal characteristics of southern plains ice storms: Insights from a regional climatology and high-resolution WRF-ARW sensitivity study. Ph.D. thesis, University of Oklahoma, $342 \mathrm{pp}$.

- L. M. Leslie, and P. J. Lamb, 2016a: Impacts of Gulf of Mexico SST anomalies on southern plains freezing precipitation: ARW sensitivity study of the 28-30 January 2010 winter storm. J. Appl. Meteor. Climatol., 55, 119-143, https://doi.org/ 10.1175/JAMC-D-14-0289.1.

,$- \ldots$, and $-2016 \mathrm{~b}$ : Synoptic pattern analysis and climatology of ice and snowstorms in the southern Great Plains, 1993-2011. Wea. Forecasting, 31, 1109-1136, https://doi.org/ 10.1175/WAF-D-15-0172.1.

NOAA, 1998: Automated Surface Observing System (ASOS) User's Guide. NOAA Tech. Rep., 61 pp., https://www.weather.gov/ media/asos/aum-toc.pdf.

Ralph, F. M., and Coauthors, 2005: Improving short-term (0-48 h) cool-season quantitative precipitation forecasting. Bull. Amer. Meteor. Soc., 86, 1619-1632, https://doi.org/10.1175/BAMS86-11-1615.

Rauber, R. M., L. S. Olthoff, M. K. Ramamurthy, and K. E. Kunkel, 2000: The relative importance of warm rain and melting processes in freezing precipitation events. J. Appl. Meteor., 39, 1185-1195, https://doi.org/10.1175/1520-0450(2000)039<1185:TRIOWR > 2.0.CO;2.

,,,--- D. Miller, and K. E. Kunkel, 2001: A synoptic weather pattern and sounding-based climatology of freezing precipitation in the United States east of the rocky Mountains. J. Appl. Meteor., 40, 1724-1747, https://doi.org/ 10.1175/1520-0450(2001)040<1724:ASWPAS > 2.0.CO;2.

Razy, A., S. M. Milrad, E. H. Atallah, and J. R. Gyakum, 2012 Synoptic-scale environments conducive to orographic impacts on cold-season surface wind regimes at Montreal, Quebec. J. Appl. Meteor. Climatol., 51, 598-616, https:// doi.org/10.1175/JAMC-D-11-0142.1.

Reeves, H. D., 2016: The uncertainty of precipitation-type observations and its effect on the validation of forecast precipitation type. Wea. Forecasting, 31, 1961-1971, https://doi.org/10.1175/ WAF-D-16-0068.1.

_ - K. L. Elmore, A. Ryzhkov, T. Schuur, and J. Krause, 2014: Sources of uncertainty in precipitation-type forecasting. Wea. Forecasting, 29, 936-953, https://doi.org/ 10.1175/WAF-D-14-00007.1.

Ressler, G. M., S. M. Milrad, E. H. Atallah, and J. R. Gyakum, 2012: Synoptic-scale analysis of freezing rain events in Montreal, Quebec, Canada. Wea. Forecasting, 27, 362-378, https://doi.org/10.1175/WAF-D-11-00071.1.

Robbins, C. C., and J. V. Cortinas, 2002: Local and synoptic environments associated with freezing rain in the contiguous
United States. Wea. Forecasting, 17, 47-65, https://doi.org/ 10.1175/1520-0434(2002)017<0047:LASEAW>2.0.CO;2.

Roebber, P. J., and J. R. Gyakum, 2003: Orographic influences on the mesoscale structure of the 1998 ice storm. Mon. Wea. Rev., 131, 27-50, https://doi.org/10.1175/1520-0493(2003) $131<0027$ :OIOTMS $>2.0 . \mathrm{CO} ; 2$.

Rutledge, S. A., and P. V. Hobbs, 1983: The mesoscale and microscale structure and organization of clouds and precipitation in midlatitude cyclones. VIII: A model for the "seederfeeder" process in warm-frontal rainbands. J. Atmos. Sci., 40, 1185-1206, https://doi.org/10.1175/1520-0469(1983)040<1185: TMAMSA $>2.0 . \mathrm{CO} ; 2$.

Ryerson, C. C., and A. C. Ramsay, 2007: Quantitative ice accretion information from the automated surface observing system. J. Appl. Meteor. Climatol., 46, 1423-1437, https://doi.org/ 10.1175/JAM2535.1.

Saha, S., and Coauthors, 2010: The NCEP Climate Forecast System Reanalysis. Bull. Amer. Meteor. Soc., 91, 1015-1057, https:// doi.org/10.1175/2010BAMS3001.1.

- and Coauthors, 2014: The NCEP Climate Forecast System version 2. J. Climate, 27, 2185-2208, https://doi.org/10.1175/ JCLI-D-12-00823.1.

Sanders, K. J., C. Gravelle, J. Gagan, and C. Graves, 2013: Characteristics of major ice storms in the central United States. J. Oper. Meteor., 1, 100-113, https://doi.org/10.15191/ nwajom.2013.0110.

, and B. L. Barjenbruch, 2016: Analysis of ice-to-liquid ratios during freezing rain and the development of an ice accumulation model. Wea. Forecasting, 31, 1041-1060, https://doi.org/ 10.1175/WAF-D-15-0118.1.

Smith, A., N. Lott, and R. Vose, 2011: The integrated surface database: Recent developments and partnerships. Bull. Amer. Meteor. Soc., 92, 704-708, https://doi.org/10.1175/2011BAMS3015.1.

Stewart, R. E., 1985: Precipitation types in winter storms. Pure Appl. Geophys., 123, 597-609, https://doi.org/10.1007/BF00877456.

_ , and P. King, 1987: Freezing precipitation in winter storms. Mon. Wea. Rev., 115, 1270-1280, https://doi.org/10.1175/ 1520-0493(1987) $115<1270$ :FPIWS > 2.0.CO;2.

Wasserstein, R. L., and N. A. Lazar, 2016: The ASA statement on p-values: Context, process, and purpose. Amer. Stat., 70, 129133, https://doi.org/10.1080/00031305.2016.1154108.

Wexler, R., R. J. Reed, and J. Honig, 1954: Atmospheric cooling by melting snow. Bull. Amer. Meteor. Soc., 35, 48-51, https:// doi.org/10.1175/1520-0477-35.2.48.

Wilks, D. S., 2011: Statistical Methods in the Atmospheric Sciences. 3rd ed. Elsevier, 676 pp.

Zerr, R. J., 1997: Freezing rain: An observational and theoretical study. J. Appl. Meteor., 36,1647-1661, https://doi.org/10.1175/ 1520-0450(1997)036<1647:FRAOAT >2.0.CO;2. 